Ricardo L. Ramirez Jr, MD

Department of Otorhinolaryngology

Head and Neck Surgery

St. Luke's Medical Center
Correspondence: Ricardo L. Ramirez Jr.,MD Department of Otorhinolaryngology Head and Neck Surgery

St. Luke's Medical Center

279 E. Rodriguez Sr. Boulevard, Quezon City, 1112

Philippines

Telefax: (632) 7230101 loc 5543

E-mail: rikrik_2006@yahoo.com

Reprints will not be available from the author.

No funding support was received for this paper. The author signed a disclosure that he has no proprietary or financial interest with any organization that may have a direct interest in the subject matter of this manuscript, or in any product used or cited in this paper.

\section{Rehabilitation of Smell After Total Laryngectomy}

Olfactory deterioration is a major consequence of total laryngectomy that results from the permanent separation of the upper and lower airways. Total laryngectomy not only affects the natural voice lost but it brings about other psychosocial problems. Leon et al.' noted that the inability to detect smoke or other odorous danger signals can threaten the personal safety of patients after laryngectomy. Impaired olfactory function adversely impacts their quality of life, which contributes to weight loss and poor nutritional status because the ability to sense different flavors requires a functional olfactory epithelium. ${ }^{1}$ The inability to detect bodily odors can cause problems in daily life and the inability to perceive agreeable odors or fragrances can be experienced as a significant loss. Since the senses called "tastes" are dependent on retronasal stimulation of the olfactory receptors, the perception of such tastes will also be negatively influenced by the loss of the sense of smell.2

Olfaction is either a passive or active process. Passive olfaction occurs during normal nasal breathing while active processes occur during smelling and sniffing. Hilgers et al. ${ }^{3}$ noted that total laryngectomy inevitably results in the loss of passive smelling and only a minority of patients are still able to actively smell anything. Their study of 63 laryngectomees found that about two thirds of the patients were anosmic and that the rest had difficulty in smelling. ${ }^{3}$

\section{Tests for olfactory acuity}

Different olfactory function tests for patients who underwent total laryngectomy have been developed in the Philippines and around the world over the past decades. The Jet Stream Olfactometer (JSO, Nagashima Medical Instruments Co., Japan) was developed as a modification of the $T$ and $T$ olfactometer (Takasago Industry, Tokyo, Japan), an olfaction test kit that includes five odorants. ${ }^{4}$ The JSO includes three of the same five odorants; odorant $\mathrm{A}$ - a dilution of $\beta$-phenyl ethyl alcohol, odorant B- a dilution of cyclotene and odorant C- a dilution of isovaleric acid. Concentrations of each odorant range over eight degrees of intensity. These odorants can be sprayed into the nasal cavity and stimulate the olfactory epithelium using the JSO apparatus. The detection threshold of each subject is defined as the lowest concentration detectable by the subject, whereas the recognition threshold is defined as the lowest concentration at which the odor can be identified. The subject's olfaction levels are classified into three groups; threshold decrease by more than degree is defined as "improved", threshold increase by more than one degree is defined as "worsened" and threshold change within \pm 1 degree is defined as "stable". 
According to the Japan Rhinology Society, the JSO is now routinely performed in Japan. ${ }^{4}$

Another test developed by Fujii et al. ${ }^{4}$ is the Alinamin test, an intravenous olfaction test that uses Alinamin, a thiol-type derivative of Vitamin B1 that smells like garlic. A dose of $10 \mathrm{mg}(2 \mathrm{ml})$ Alinamin is injected into the median vein of the left arm at a constant rate over 20 s. The latency and duration are measured. The latency interval is the time until recognition of smell while duration is the time from recognition until disappearance of the smell. The usual latency interval is 7-8 seconds and the duration is 1-2 minutes. In laryngectomees, the use of nasal plugs during testing prevents breathing the garlic smell from the trachea-stoma. ${ }^{4}$

Leon et al. ${ }^{1}$ also developed olfactory function tests for post laryngectomy patients which utilise their orthonasal and retronasal olfaction. Orthonasal olfaction tests include the Butanol threshold test and odor identification test using common odors developed at the Connecticut Chemosensory Clinical Research Center (CCCRC). In the Butanol threshold test, two identical plastic bottles, one containing water and the other containing dilute concentration of butanol are simultaneously presented to the subject. Subjects are instructed to occlude one nostril and place the tip of the bottle immediately beneath the other nostril. The bottles are then squeezed at least twice for the odorants to reach the olfactory epithelium. The subject has to choose which of the bottles contain something other than water. The odor identification test uses common household odorants like peanut butter, Ivory ${ }^{\mathrm{TM}}$ soap, mothballs, Vicks ${ }^{\mathrm{TM}}$ vaporub, chocolate, baby powder, coffee and cinnamon which are placed in opaque squeezable bottles. The odorants are delivered by squeezing the bottle underneath one nostril while the other nostril is occluded. The subjects then choose from a printed list containing the correct items as well as an equal number of distractor items. Possible scores range from 0 to 7 items correctly identified and scores of both nostrils are averaged to arrive at the final scores. The scores from the butanol threshold test and identification tests are subsequently averaged to arrive at a composite score for the orthonasal olfactory ability. ${ }^{1}$

Retronasal olfactory function testing involves different flavoured powders applied on the tongue and then identified using a forcedchoice paradigm. Twenty (20) flavoured powders purchasable at a grocery store include garlic, strawberry, milk, lemon, orange, vanilla, cinnamon, cloves, paprika, curry, butter buds, bread, cocoa, celery, chicken bouillon, grape, raspberry, onion, ginger and coffee. A small amount of each flavoured powder is scooped up into the tip of a disposable straw and deposited on the subject's mid-dorsal tongue. The subject chooses from a printed list of 4 items for each test. Answers are scored from 0 to 20 correct item identifications. ${ }^{1}$

Risberg-Berlin et al. used the Scandinavian Odor Identification Test (SOIT) in determining the olfactory function of post-larygectomy Swedish patients. ${ }^{5}$ The test involves 16 different odors and 4 alternative responses per odor to choose from. The smell is generated by $5 \mathrm{ml}$ of odor stimulus placed in a $10 \mathrm{ml}$ glass jar. The test has age and sex related cutoff scores and categorizes patients as smellers or non-smellers. Smellers are subjects diagnosed with functional hyposmia and normosmia while non-smellers are subjects diagnosed with anosmia.

Ramirez et al. subjectively and objectively evaluated the olfactory function of Filipino post-laryngectomy patients using a questionnaire adapted from that of the Smell and Taste Clinic of Hospital of University Pennsylvania (HUP) and the Santo Tomas Smell Identification Test respectively. ${ }^{6}$ Each subject is asked to assess their olfactory function and the impact of such on their daily lives including questions on olfactory disorder prior to laryngectomy, sense of smell immediately after laryngectomy, present sense of olfaction, degree of change in olfaction and smell condition after olfactory rehabilitation. Olfactory function of each subject is evaluated using the Santo Tomas Smell Identification Test (ST-SIT) ${ }^{7}$ that uses 45 odorants, each enclosed in an opaque polyethylene squeeze bottle. Each odorant is smelled by the subject and identified from a written list of choices. The odorants have a corresponding score and the summation of these scores serves to discriminate those with normal olfactory function from those who are hyposmic and anosmic. Based on the ST-SIT, normal individuals score more than 90; hyposmic individuals score 70 to 90 while anosmics score less than 70 .

\section{Intervention}

Different techniques have been developed for improvement of olfaction in post laryngectomy patients. Schawrtz ${ }^{8}$ tried to improve smell by using a "larynx bypass," in which airflow from the stoma is directed to the mouth, creating an artificial airflow in the nose. However, this method is troublesome and impractical for use in everyday life. ${ }^{8}$ Techniques like"glossopharyngeal press,", "buccopharyngeal maneuver" and "buccopharyngeal sniffing" have also been described in different olfactory rehabilitation studies. These are less obtrusive methods that generate some degree of nasal airflow by creating volume changes in the pharynx with the mouth closed, but these are not widely applied.

Hilgers ${ }^{2}$ and Ramirez et al. ${ }^{10}$ use the nasal airflow-inducing maneuver to restore nasal airflow by creating underpressure in the oral cavity and oropharynx by having the patient perform an extended yawning movement while keeping the lips securely closed and simultaneously lowering the jaw, floor of the mouth, tongue, base of tongue and soft 
PRACTICE PEARLS

palate. The underpressure created generates nasal airflow allowing odorant molecules to reach the olfactory epithelium. The movement is repeated rapidly several times to increase its effectiveness but the technique requires a training period and constant use.

\section{Conclusion}

Olfactory impairment is common in patients who have undergone laryngectory. Different rehabilitation programs address the olfactory deficit of post-laryngectomy patients and aim to prevent or decrease the negative impact on quality of life that loss of olfaction brings. Aside from voice and pulmonary rehabilitation, olfactory rehabilitation should routinely be incorporated in the rehabilitation program of postlaryngectomy patients.

\section{REFERENCES}

1. Leon EA, Catalanotto FA, Werning JW. Retronasal and orthonasal olfactory ability after laryngectomy. Arch Otolaryngology Head and Neck Surg 2007; 133:32-36.

2. Hilgers FJM, van Dam FSAM, Keyzers S, Koster MN, van As C, Muller MJ. Rehabilitation of olfaction after laryngectomy by means of a nasal airflow-inducing maneuver. Arch Otolaryngology Head and Neck Surg 2000; 126: 726-732.

3. van Dam FSAM, Hilgers FJM, Emsbroek G, Touw Fl, van As CJ, De Jong N . Deterioration of olfaction and gestation as a consequence of total laryngectomy. Laryngoscope. 1999; 109: 1150-1155

4. Fujii M, Fukazawa K, Hatta C, Yasuno H, Sakagami M. Olfactory acuity after total laryngectomy Chemical Senses 2002; 27: 117-121.

5. Risberg-Berlin B, Ryden A, Moller RY, Finizia C. Effects of total laryngectomy on olfactory function, health-ralated quality of life and communication: a 3 year follow up study. BMC Ear Nose and Throat Disorders 2009; 9:8.

6. Ramirez RL, Panganiban WD, Romualdez JA. Subjective and objective assessment of olfactory function in post-laryngectomy patients. Philipp J Otolaryngol Head Neck Surg 24(2): 19-22 December 2009.

7. David J, Campomanes B, Dalupang J, Loberiza F. Smell identification test. Philipp J Otolaryngol Head Neck Surg 1994;62-8

8. Schwartz DN, Mozell MM, Youngentob SL, Leopold DL, Sheehe PR. Improvement of olfaction in laryngectomized patients with the larynx bypass. Laryngoscope. 1987; 97:1280-1286.

9. Risberg-Berlin B, Ylitalo R, Finizia C. Screening and rehabilitation of olfaction after tota laryngectomy in Swedish patients: results from intervention study using the nasal airflowinducing maneuver. Arch Otolaryngology Head and Neck Surg. 2006; 132 (3): 301-306.

10. Ramirez RL, Romualdez JA, Casile R. The use of nasal airflow inducing maneuver in olfactory rehabilitation of post laryngectomy patients. Unpublished manuscript presented at Descriptive Research Contest ( $2^{\text {nd }}$ Place) Philippine Society of Otolaryngology-Head and Neck Surgery, Congo Grill Restaurant, September 2008. 